Ann. Zootech., I976, 25 (2), 273-27().

NOTE

\title{
RÉPARTITION DES MASSES MUSCULAIRES CHEZ LE JEUNE BOVIN MÂLE ENTIER, ET SON ÉVOLUTION AU COURS DE LA PÉRIODE D'ENGRAISSEMENT ENTRE 8-9 ET 16-17 MOIS
}

\author{
J. ROBBELIN et Y. GEAY \\ avec la collaboration technique de R. JAILLER et R. JAII.IER \\ Laboratoire de la Production de Viande, \\ Centre de Recherches de Clermond-Ferrand, I. N.R. A., \\ Theix, Saint Genès Champanelle, \\ 63110 Beaumont
}

RÉSUMÉ

Nous avons mesuré la croissance relative des différentes régions musculaires de 3 I jeunes bovins mâles entiers, de deux races (Charolaise et Limousine) entre 8 et $\mathbf{1} 7$ mois. Los muscles du collier et du thorax ont une croissance relative plus élevée $(b>1,20)$ que celle des muscles du membre postérieur et du dos $(b<0,90)$. Les autres régions musculaires ont une croissance relative voisine de celle de l'ensemble de la musculature

Dans une récente étude (RoBelix ct al., 1974), nous avons clécrit l'évolution de la répartition des différents tissus de la carcasse des taurillons Frisons. Nous avons également abordé l'étude du développenent des régions corporelles; mais nos conclusions, relatives à une seule race, ont été limitées par le fait que nous n'avions pas mesuré le poids cles différents composants tissulaires de chaque région et que ces régions de la carcasse correspondaient à une découpe de demi-gros qui n'a pas de valeur générale.

Depuis, nous avons entrepris une étude plus précise sur le développement de la musculature des jeunes bovins ; la musculature a été divisée en plusieurs régions correspondant à un ensemble de muscles bien défini (cf. Annexe) pesé après dissection de la carcasse. Des études analogues ont déjà été réalisées sur les lapins (Vezinhet et al, r972), les ovins (Lhose et al, I97I ; Boccard et Dumont, 1973) et les bovins mâles castrés de races Anglosaxonnes (Butterfield et Berg, 
I966; SEEBECK et TULLOH, I968), mais jamais à notre connaissance, sur des jeunes bovins mâles entiers de races Françaises. Nous rapportons ici, les premiers résultats que nous avons obtenus à partir de la dissection de $3 \mathrm{I}$ animaux dont les caractéristiques sont décrites au tableau $\mathrm{I}$.

TABLEAU I

Caractéristiques des animaux utilisés

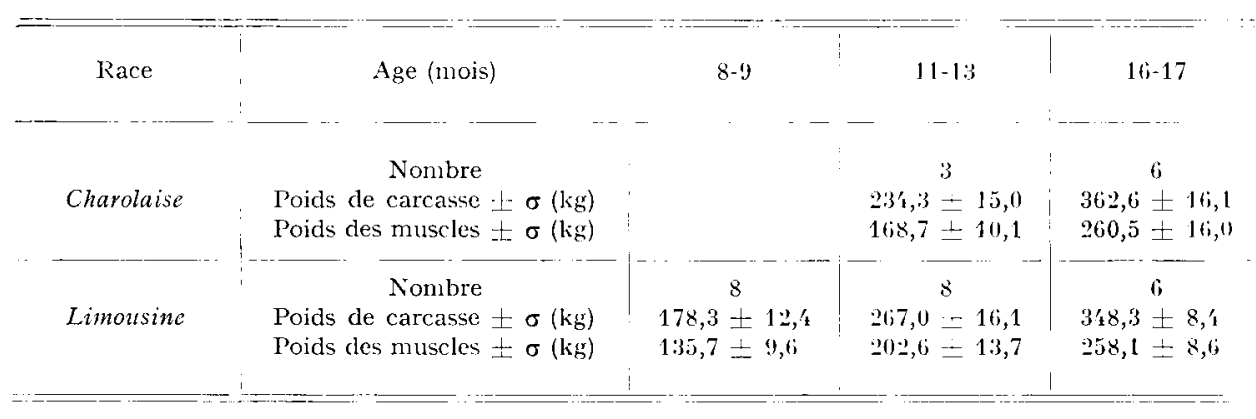

Les carcasses de ces animaux ont été disséquées et nous avons mesuré le poids des muscles des différentes régions. Nous avons utilisé l'équation de Huxley $: \log \mathrm{Y}=b \log \mathrm{X}+$ a pour décrire la relation entre le poids des différentes masses musculaires $(Y)$, et le poids des muscles de la carcasse (X). L'analyse de covariance n'a pas permis de mettre en évidence de différence significative entre les races dans les pentes $(b)$ et les moyennes ajustécs; nous avons donc réalisé lá suite des calculs sur la totalité des animaux.

Des résultats rapportés au tablcau 2, on peut retenir quelques constatations concernant la liaison entre le poids des muscles des différentes régions et le poids total de la musculature, la répartition pondérale moyenne des différentes masses musculaires, et son évolution au cours de la période d'engraissement entre 8-9 et 16-17 mois.

Le poids des muscles des différentes régions est fortement lié ( $R^{2}$ compris entre 0,83 et 0,97 ) au poids total de la musculature. Lorsque le poids total de la musculature est connu (ou fixé), on peut déterminer le poids des différentes régions musculaires avec une erreur résiduelle comprise entre 3,9 et $\mathrm{I} 3,5 \mathrm{p}$. Ioo du poids de chaque région (tabl. 2). La répartition des masses musculaires semble indépendante de la conformation et en particulier de la compacité de la cuisse (tabl. 2), telle qu'elle a été définie par Dumoxi et al. (196I). Ainsi, les critères de compacité sont reliés à la quantité totale de muscles (GEAy et Beranger, 1969), mais sont indépendants ( $\mathrm{P}<0,0 \mathrm{o}$ ) de la répartition des masses musculaires. Ces résultats sont en accord avec ceux de Dumont it al (I96I) chez les bovins, et Boccard et Dumont (I969) chez les ovins.

Les muscles de la région dorsale et du membre postérieur, parmi lesquels se trouvent les muscles les plus tendres, représentent une part importante $\left(4^{8,5} \mathrm{p}\right.$. IOo) de la musculature. Cependant, quelle que soit la région considérée, son importance dans la musculature totale varie avec le poids de la musculature. $D^{\prime}$ après la valeur des coefficients de régression, la part des muscles clu thorax et du collier a tendance à augmenter tandis que celle des muscles de la région dorsale, et surtout du membre postérieur, diminue lorsque le poids total de la musculature augmente (tabl. 2). Ces résultats confirment ceux que nous avons obtenus sur la croissance relative de la chair (muscles + dépôts adipcux) cles différentes régions chez le taurillon Frison (RoBelin et ul., 1974). La plupart des coefficients de régression sont voisins de ceux qui ont été obtenus par SeEbeck et Tulloh (1968) avec des boufs de race Angus. Cependant, on remarque une croissance relative des muscles du collier beaucoup plus élevée chez les taurillons $(b=\mathrm{I}, 30$ ) que chez les bœufs. Pour un même prids total de muscles $(254 \mathrm{~kg})$, les muscles de la région cervicale représcntent une plus grande proportion de la musculature totale sur les taturillons de race Charolaise 
ou Limonsine (i 7, I p. 100), que sur les boufs de race Chavolaise (13,6 p. 100); (I)Umont et al., I961). Inversement, les muscles du membre postérieur représentent une part plus faible sur les

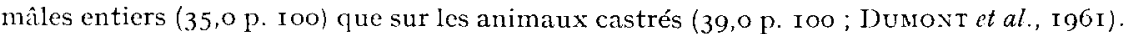

TABLEAU 2

Répartition pondérale moyenne des masses musculaires de la carcasse des jeunes bovins (mâles entiers) et évolution de cette répartition

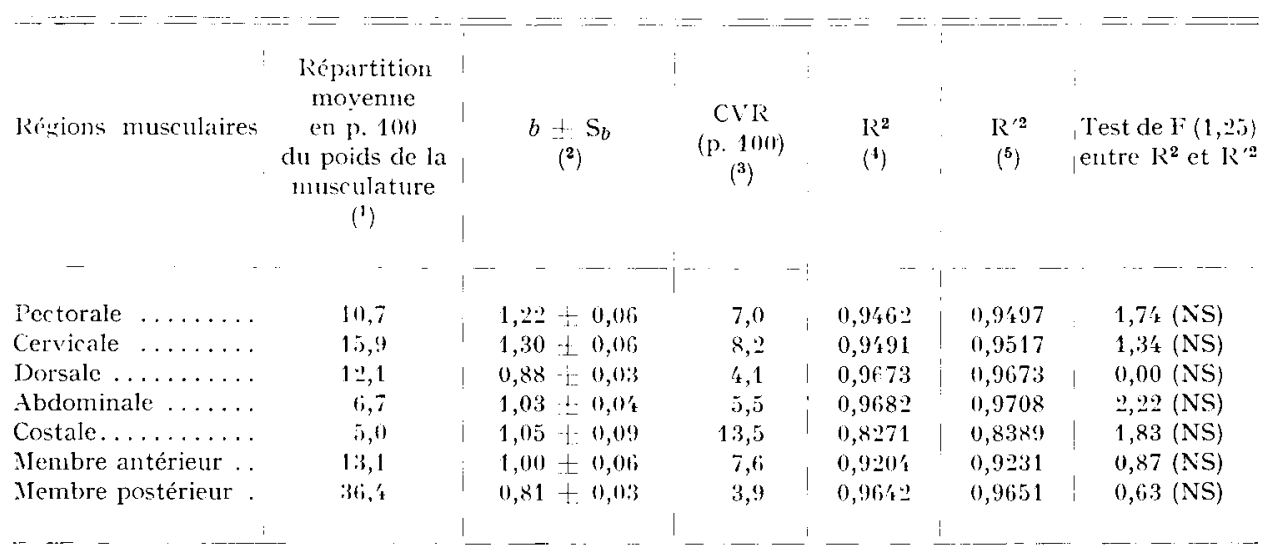

(1) Répartition pondérale moyenne, calculée pour un poids moyen de la musculature de $202,9 \mathrm{~kg}$.

${ }^{(2)}$ Coefficient de régression du poids des muscles d'une région (Y) par rapport au poids total de la mulsculature $\left(\mathrm{X}_{1}\right): \log \mathrm{Y}=b \log \mathrm{X}_{1}+a . \mathrm{S}_{b}$ est l'écart-type de $b$.

(3) Écart-type résiduel de la régression de $\mathrm{Y}$ sur $\mathrm{X}_{1}$ exprimé en pourcentage de $\mathrm{Y}$.

(4) $R^{2}$ est le carré du coefficient de corrélation entre $\log Y$ et $\log \mathrm{X}_{1}$.

(5) $R^{\prime 2}$ est le carré du coefficient de corrélation multiple entre $\log Y$ d'une part, $\log \mathrm{X}_{1}$ et $\log \mathrm{X}_{2}$ (compacité de la cuisse) d'autre part.

Ces premiers résultats apportent de nouvelles informations sur l'évolution de la composition corporelle des jeunes bovins mâles entiers de races Françaises pendant la période d'engraissement. De nos résultats antérieurs et de ceux qui sont présentés ici, on peut déjà retenir que les régions postérieures et dorsales n'ont pas un développement rapide chez le jeune bovin mâle entier au cours de la période d'engraissement entre 8-9 et i6-I7 mois. Plus l'animal sera abattu lourd et âgé, plus la proportion d' "avant " et en particulier de "collier "sera élevée dans la carcasse.

Reçu pour publicalion en décembre 1975.

\section{SUMMARY}

DISTRIBUTION AND GROWTH PATTERNS OF MUSCLE MASSES IN YOUNG BULLS DURING FATTENING BETWEEN 8-9 AND I6-I7 MONTHS

The relative growth patterns of the different groups of muscles in $3 \mathbf{I}$ carcasses of young bulls from 2 breeds (Chavolais, Limousin), between 8 to I 7 months of age were measured. According to the data collected, the muscles of the neck and thorax showed a higher relative growth $(b>$ r.20) than those of the hind leg and the back $(b<0.90)$. The other muscles exhibited almost the same growth rate than that of the whole musculature. 


\section{RÉFÉRENCES BIBLIOGRAPHIQUES}

Boccard R., Demont 13. L., Ig60. litude de la production de viande chez les ovins. II. Variation de l'importance relative des différentes régions corporelles de l'agneau de boucherie. Ann. Zootech., 9, $355-363$.

Boccard R., Dumoni 13. L, r973. Iitude de la production de viande chez les ovins. IX. Variations de l'organisation de la musculature de l'Agneau en fonction de la vitesse de croissance. Ann. Zootech., 22, 423-43I.

Boccard R., Demont 13, L., Lefebvre J., I962. litude de la production de viande chez les ovins. V. Note sur la croissance relative des régions corporelles de l'A gneall. Ann. Zootech., 11, $257-262$.

Butterfield R. M., BlRG R. T., I966. Relative growth patterns of commercially important muscle groups of cattle. Res. Vet. Sci, 7, 389-393.

Dunont B. L., Le Guelte P., Arnoux J., ig6r. Étude biométrique des bovins de boucherie. I. Variabilité de la composition ana tomique de la carcasse des bovins Charolais. Ann. Zootech., 10, I49-I54.

Geay Y., Beranger C., I969. Estimation de la composition de la carcasse de jeunes bovins à partir de la composition d'un morceau monocostal prélevé au niveau de la I I côte. Ann. Zootech., 18,65-77.

Lohse C. L., Moss F. P., Butterfield R. I., rg7x. Growth patterns of nuscles of merino sheep from birth to 5 I 7 days. Anim. Prod., 13, I I7-I26.

Robelin J., Geax Y., Beranger C., I974. Croissance relative des différents tissus, organes et régions: corporelles des taurillons Frisons, durant la phase d'engraissement de 9 à is mois. Ann. Zootech., 23, $313-323$.

SEEBECK R. M., Trelor N. M., I968. Ievelopmental growth and body weight loss of cattle, III. Dissected components of the commercially dressed carcass, following anatomical boundaries. A $u s t$.. J. A gric. Res., 19, $673-88$.

Vezinhet A., Rouvier R,, Lulor J. P., Cantier J., ig72. Allométrie de croissance chez le lapin III. principales régions du système musculaire. Ann. Biol. anim. Bioch. Biophys., 12, 33-45.

\section{ANNEXE}

\section{RÉPARTITION DES MUSCI,ES DE IA CARCASSE \\ DANS LES DIFFÉRENTES RÉGION'S MUSCUIAIRES}

Région Corvicale

sternocéphalique

brachiocéphalique

sternohyoïdicn

sternothyrö̈lien

omohyoïdien

scalène

trapèze

omotransversaire

rhomboïde

splénius

dentclé du cou

grand complexus

petit complexus

épineux du cou

(coupé au niveau de la I ${ }^{\text {re }}$ côte, sternocephalicus

brachiocephalicus

sternohyoideus

sternothyroideus

omohyoideus

scaleni

trapezius

omotransversarius

rhomboideus

splenius

serratus ventralis cervicis

semi spinalis capitis

longissimus atlantis et capitis

interspinales cervicis 